\title{
Article
}

\section{Sandwich type results for meromorphic functions with respect to symmetrical points}

\author{
Kuldeep Kaur Shergill ${ }^{1, *}$ and Sukhwinder Singh Billing ${ }^{1}$ \\ 1 Department of Mathematics, Sri Guru Granth Sahib World University Fatehgarh Sahib-140407(Punjab), India. \\ * Correspondence: kkshergill16@gmail.com
}

Academic Editor: Teodor Bulboaca

Received: 25 November 2021; Accepted: 13 December 2021; Published: 24 December 2021.

\begin{abstract}
In the present paper, we use the technique of differential subordination and superordination involving meromorphic functions with respect to symmetric points and also derive some sandwich results. As a consequence of main result, we obtain results for meromorphic starlike functions with respect to symmetrical points.
\end{abstract}

Keywords: Meromorphic function; Meromorphic starlike function; Differential subordination; Superordination.

MSC: 30C45; 30C80.

\section{Introduction}

$\mathbf{L}$

et $\Sigma$ denote the class of functions of the form

$$
f(z)=\frac{1}{z}+\sum_{k=1}^{\infty} a_{k} z^{k-1}
$$

which are analytic in the punctured unit disc $\mathbb{E}_{0}=\mathbb{E} \backslash\{0\}$, where $\mathbb{E}=\{z \in \mathbb{C}:|z|<1\}$. A function $f \in \Sigma$ is said to be meromorphic starlike of order $\alpha$ if $f(z) \neq 0$ for $z \in \mathbb{E}_{0}$ and

$$
-\Re\left(\frac{z f^{\prime}(z)}{f(z)}\right)>\alpha, \quad(\alpha<1 ; z \in \mathbb{E}) .
$$

The class of such functions is denoted by $\mathcal{M S}^{*}(\alpha)$ and write $\mathcal{M S}^{*}=\mathcal{M S}^{*}(0)$-the class of meromorphic starlike functions.

In 1959, Sakaguchi [1] introduced and studied the class of starlike functions with respect to symmetric points in $\mathbb{E}$. Further investigations into the class of starlike functions with respect to symmetric points can be found in $[2,3]$.

Recently, Ghaffar et al., [4] introduced and investigated a class of meromorphic starlike functions with respect to symmetric points which satisfies the condition

$$
-\frac{2 z f^{\prime}(z)}{f(z)-f(-z)} \prec \frac{1+A z}{1+B z}, z \in \mathbb{E}_{0}
$$

and $-1 \leq B<A \leq 1$. We denote the above class by $\mathcal{M S}^{s *}[A, B]$. If $f$ is analytic and $g$ is analytic univalent in open unit disk $\mathbb{E}$, we say that $f(z)$ is subordinate to $g(z)$ in $\mathbb{E}$ and written as $f(z) \prec g(z)$ if $f(0)=g(0)$ and $f(\mathbb{E}) \subset g(\mathbb{E})$. To derive certain sandwich-type results, we use the dual concept of differential subordination and superordination.

Let $\Phi: \mathbb{C}^{2} \times \mathbb{E} \longrightarrow \mathbb{C}(\mathbb{C}$ is the complex plane) and $h$ be univalent in $\mathbb{E}$. If $p$ is analytic in $\mathbb{E}$ and satisfies the differential subordination

$$
\Phi\left(p(z), z p^{\prime}(z) ; z\right) \prec h(z), \Phi(p(0), 0 ; 0)=h(0),
$$


then $p$ is called a solution of the differential subordination (1). The univalent function $q$ is called a dominant of differential subordination (1) if $p \prec q$ for all $p$ satisfying (1). A dominant $\tilde{q} \prec q$ for all dominants $q$ of (1), is said to be the best dominant of $(1)$.

Let $\Psi: \mathbb{C}^{2} \times \mathbb{E} \longrightarrow \mathbb{C}\left(\mathbb{C}\right.$ is the complex plane) be analytic and univalent in domain $\mathbb{C}^{2} \times \mathbb{E}, h$ be analytic in $\mathbb{E}, p$ is analytic and univalent in $\mathbb{E}$, with $\left(p(z), z p^{\prime}(z) ; z\right) \in \mathbb{C}^{2} \times \mathbb{E}$ for all $z \in \mathbb{E}$. Then $p$ is called a solution of first order differential superordination if it satisfies

$$
h(z) \prec \Psi\left(p(z), z p^{\prime}(z) ; z\right), h(0)=\Psi(p(0), 0 ; 0)
$$

An analytic function $q$ is called a subordinant of differential superordination (2) if $q \prec p$ for all $p$ satisfying (2). A univalent subordinant $\tilde{q}$ that satisfies $q \prec \tilde{q}$ for all subordinants $q$ for (2), is said to be the best subordinant of (2).

In this paper we study the concepts of subordination and superordination to obtain meromorphic starlikeness with respect to symmetric points.On the basis of the theory we also investigate some important sandwich results of symmtric meromorphic functions.

\section{Preliminaries}

We shall use the following lemmas to prove our result.

Lemma 1. [5] Let $q$ be univalent in $\mathbb{E}$ and let $\theta$ and $\phi$ be analytic in a domain $\mathbb{D}$ containing $q(\mathbb{E})$, with $\phi(w) \neq 0$, when $w \in q(\mathbb{E})$. Set $Q_{1}(z)=z q^{\prime}(z) \phi[q(z)], h(z)=\theta[q(z)]+Q_{1}(z)$ and suppose that either

(i) $h$ is convex, or

(ii) $Q_{1}$ is starlike.

In addition, assume that

(iii) $\Re\left(\frac{z h^{\prime}(z)}{Q_{1}(z)}\right)>0$ for all $z$ in $\mathbb{E}$.

If $p$ is analytic in $\mathbb{E}$, with $p(0)=q(0), p(\mathbb{E}) \subset \mathbb{D}$ and

$$
\theta[p(z)]+z p^{\prime}(z) \phi[p(z)] \prec \theta[q(z)]+z q^{\prime}(z) \phi[q(z)], z \in \mathbb{E},
$$

then $p(z) \prec q(z)$ and $q$ is the best dominant.

Definition 1. We denote by $Q$ the set of functions $p$ that are analytic and injective on $\overline{\mathbb{E}} \backslash \mathbb{B}(p)$, where

$$
\mathbb{B}(p)=\left\{\zeta \in \partial \mathbb{E}: \lim _{z \rightarrow \zeta} p(z)=\infty\right\},
$$

and are such that $p^{\prime}(\zeta) \neq 0$ for $\zeta \in \partial \mathbb{E} \backslash \mathbb{B}(p)$.

Lemma 2. [6] Let $q$ be the univalent in $\mathbb{E}$ and let $\theta$ and $\phi$ be analytic in a domain $\mathbb{D}$ containing $q(\mathbb{E})$. Set $Q_{1}(z)=$ $z q^{\prime}(z) \phi[q(z)], h(z)=\theta[q(z)]+Q_{1}(z)$ and suppose that $(i) Q_{1}(z)$ is starlike in $\mathbb{E}$; and $(i i) \Re\left(\frac{\theta^{\prime}(q(z))}{\phi(q(z))}\right)>0$, for $z \in \mathbb{E}$. If $p \in \mathcal{H}[q(0), 1] \cap Q$, with $p(\mathbb{E}) \subset \mathbb{D}$ and $\theta[p(z)]+z p^{\prime}(z) \phi[p(z)]$ is univalent in $\mathbb{E}$ and

$$
\theta[q(z)]+z q^{\prime}(z) \phi[q(z)] \prec \theta[p(z)]+z p^{\prime}(z) \phi[p(z)], z \in \mathbb{E},
$$

then $q(z) \prec p(z)$ and $q$ is the best subordinant.

Lemma 3. [7] The function $q(z)=\frac{1}{(1-z)^{2 a b}}$ is univalent in $E$ if and only if $|2 a b-1| \leq 1$ or $|2 a b+1| \leq 1$. 


\section{Subordination results}

Theorem 1. Let $q$ be univalent in $\mathbb{E}$, with $q(0)=1$, and let

$$
\Re\left(1+\frac{z q^{\prime \prime}(z)}{q^{\prime}(z)}\right)>\max \left\{0 ;-\Re \frac{1}{\gamma}\right\}, z \in \mathbb{E},
$$

where $\gamma \in \mathbb{C}^{*}:=\mathbb{C} \backslash\{0\}$. If $f \in \Sigma$ satisfy the condition

$$
f(z) \neq f(-z), z \in \mathbb{E}_{0}
$$

and

$$
\frac{-2(1+\lambda) z f^{\prime}(z)}{f(z)-f(-z)}+\frac{2 \lambda z^{2} f^{\prime}(z)\left(f^{\prime}(z)+f^{\prime}(-z)\right)}{(f(z)-f(-z))^{2}}-\frac{2 \lambda z^{2} f^{\prime \prime}(z)}{f(z)-f(-z)} \prec q(z)+\lambda z q^{\prime}(z)
$$

then

$$
\frac{-2 z f^{\prime}(z)}{f(z)-f(-z)} \prec q(z)
$$

and $q$ is the best dominant of (5).

Proof. Setting

$$
p(z)=\frac{-2 z f^{\prime}(z)}{f(z)-f(-z)}, z \in \mathbb{E},
$$

from assumption (4) it follows that $p$ is analytic in $\mathbb{E}$, with $p(0)=1$. A simple computation shows that

$$
\frac{-2(1+\lambda) z f^{\prime}(z)}{f(z)-f(-z)}+\frac{2 \lambda z^{2} f^{\prime}(z)\left(f^{\prime}(z)+f^{\prime}(-z)\right)}{(f(z)-f(-z))^{2}}-\frac{2 \lambda z^{2} f^{\prime \prime}(z)}{f(z)-f(-z)}=p(z)+\lambda z p^{\prime}(z),
$$

hence, the subordination (5) is equivalent to $p(z)+\lambda z p^{\prime}(z) \prec q(z)+\lambda z q^{\prime}(z)$.

Now, in order to prove our result we will use Lemma 1. Consider the functions $\theta(w)=w$ and $\phi(w)=\gamma$ analytic in $\mathbb{C}$, and set

$$
Q(z)=z q^{\prime}(z) \phi(q(z))=\gamma z q^{\prime}(z) \text { and } h(z)=\theta(q(z))+Q(z)=q(z)+\gamma z q^{\prime}(z) .
$$

Since $Q(0)=0$ and $Q^{\prime}(0)=\gamma q^{\prime}(0) \neq 0$, the assumption (3) implies that $Q$ is starlike in $\mathbb{E}$ and

$$
\Re\left(\frac{z h^{\prime}(z)}{Q(z)}\right)=\Re\left(1+\frac{z q^{\prime \prime}(z)}{q^{\prime}(z)}+\frac{1}{\gamma}\right)>0, z \in \mathbb{E} .
$$

Therefore, Lemma 1 and assumption (5) imply $p(z) \prec q(z)$ and the function $q$ is the best dominant of (5).

If we take $q(z)=\frac{1+A z}{1+B z},-1 \leq B<A \leq 1$ in Theorem 1 then the condition (3) reduces to

$$
\Re \frac{1-B z}{1+B z}>\max \left\{0 ;-\Re \frac{1}{\lambda}\right\}, z \in \mathbb{E}
$$

It is easy to see that the function $\chi(\zeta)=\frac{1-\zeta}{1+\zeta}$ with $|\zeta|<|B|$ is convex in $E$ and since $\chi(\bar{\zeta})=\overline{\chi(\zeta)}$ for all $|\zeta|<|B|$, it follows that $\chi(\mathbb{E})$ is a convex domain symmetric with respect to the real axis. Hence

$$
\inf \left(\Re \frac{1-B z}{1+B z}: z \in \mathbb{E}\right)>\frac{1-|B|}{1+|B|}>0
$$

Thus, the inequality (6) is equivalent to $\Re \frac{1}{\lambda} \geq \frac{|B|-1}{|B|+1}$, hence we deduce the following corollary: 
Corollary 1. Let $\lambda \in \mathbb{C}^{*}$ and $-1 \leq B<A \leq 1$ with $\frac{1-|B|}{1+|B|} \geq \max \left\{0 ;-\Re \frac{1}{\lambda}\right\}$. If $f \in \Sigma$ satisfy the condition (4) and

$$
\frac{-2(1+\lambda) z f^{\prime}(z)}{f(z)-f(-z)}+\frac{2 \lambda z^{2} f^{\prime}(z)\left(f^{\prime}(z)+f^{\prime}(-z)\right)}{(f(z)-f(-z))^{2}}-\frac{2 \lambda z^{2} f^{\prime \prime}(z)}{f(z)-f(-z)} \prec \frac{1+A z}{1+B z}+\lambda \frac{(A-B) z}{(1+B z)^{2}},
$$

then

$$
\frac{-2 z f^{\prime}(z)}{f(z)-f(-z)} \prec \frac{1+A z}{1+B z}
$$

i.e., $f \in \mathcal{M S}^{s *}[A, B]$. Moreover, the function $\frac{1+A z}{1+B z}$ is the best dominant of (8).

For $A=1$ and $B=-1$, the above corollary reduces to the next special case:

Remark 1. Let $\lambda \in \mathbb{C}^{*}$ with $\Re \frac{1}{\lambda} \geq 0$. If $f \in \Sigma$ satisfy the condition (4) and

$$
\frac{-2(1+\lambda) z f^{\prime}(z)}{f(z)-f(-z)}+\frac{2 \lambda z^{2} f^{\prime}(z)\left(f^{\prime}(z)+f^{\prime}(-z)\right)}{(f(z)-f(-z))^{2}}-\frac{2 \lambda z^{2} f^{\prime \prime}(z)}{f(z)-f(-z)} \prec \frac{1+z}{1-z}+\lambda \frac{2 z}{(1-z)^{2}},
$$

then

$$
\frac{-2 z f^{\prime}(z)}{f(z)-f(-z)} \prec \frac{1+z}{1-z^{\prime}}
$$

i.e., $f \in \mathcal{M S}^{\mathcal{S}}$, or $f$ is meromorphic starlike with respect to symmetrical points in $\mathbb{E}$. Moreover, the function $\frac{1+z}{1-z}$ is the best dominant of (9).

Theorem 2. Suppose that $q$ be univalent in $\mathbb{E}$ with $q(0)=1$ and $q(z) \neq 0$ for all $z \in \mathbb{E}$ such that

$$
\Re\left(1+\frac{z q^{\prime \prime}(z)}{q^{\prime}(z)}-\frac{z q^{\prime}(z)}{q(z)}\right)>0, z \in \mathbb{E},
$$

where $\gamma, \mu \in \mathbb{C}^{*}$ and $v, \eta \in \mathbb{C}$ with $v+\eta \neq 0$ and let $f \in \Sigma$ satisfy the conditions

$$
v z\left(f^{\prime}(z)+f^{\prime}(-z)\right)+\eta(f(z)-f(-z)) \neq 0, z \in \mathbb{E}_{0}
$$

and

$$
\frac{-2(v+\eta) z f^{\prime}(z)}{v z\left(f^{\prime}(z)+f^{\prime}(-z)\right)+\eta(f(z)-f(-z))}, z \in \mathbb{E}_{0}
$$

If

$$
1+\gamma \mu\left[1-\frac{v z\left[z\left(f^{\prime}(z)+f^{\prime}(-z)\right)\right]^{\prime}+\eta z\left(f^{\prime}(z)+f^{\prime}(-z)\right)}{v z\left(f^{\prime}(z)+f^{\prime}(-z)\right)+\eta(f(z)-f(-z))}+\frac{z f^{\prime \prime}(z)}{f^{\prime}(z)}\right] \prec 1+\gamma \frac{z q^{\prime}(z)}{q(z)},
$$

then

$$
-\left[\frac{2(v+\eta) z f^{\prime}(z)}{v z\left(f^{\prime}(z)+f^{\prime}(-z)\right)+\eta(f(z)-f(-z))}\right]^{\mu} \prec q(z)
$$

and $q$ is the best dominant of (13) (the power is the principal one).

Proof. Define a function

$$
p(z)=-\left[\frac{2(v+\eta) z f^{\prime}(z)}{v z\left(f^{\prime}(z)+f^{\prime}(-z)\right)+\eta(f(z)-f(-z))}\right]^{\mu}, z \in \mathbb{E} .
$$

According to the assumptions (11) and (12), the multivalued power function $p$ has an analytic branch in $\mathbb{E}$ with $p(0)=1$ and from the (14) it follows that

$$
\mu\left[1-\frac{v z\left[z\left(f^{\prime}(z)+f^{\prime}(-z)\right)\right]^{\prime}+\eta z\left(f^{\prime}(z)+f^{\prime}(-z)\right)}{v z\left(f^{\prime}(z)+f^{\prime}(-z)\right)+\eta(f(z)-f(-z))}+\frac{z f^{\prime \prime}(z)}{f^{\prime}(z)}\right]=\frac{z p^{\prime}(z)}{p(z)} .
$$


To prove our desired result we will use Lemma 1. Thus, let the functions $\theta(w)=1$ and $\phi(w)=\frac{\gamma}{w}$. Also, if we let $Q(z)=z q^{\prime}(z) \phi(q(z))=\gamma z q^{\prime}(z)$ and $h(z)=\theta(q(z))+Q(z)=1+\gamma \frac{z q^{\prime}(z)}{q(z)}$.

Since $Q(0)=0$ and $Q^{\prime}(0)=\gamma q^{\prime}(0) \neq 0$, the assumption (10) implies that $Q$ is starlike in $\mathbb{E}$ and

$$
\Re\left(\frac{z h^{\prime}(z)}{Q(z)}\right)=\Re\left(1+\frac{z q^{\prime \prime}(z)}{q^{\prime}(z)}+\frac{z q^{\prime}(z)}{q(z)}\right)>0, z \in \mathbb{E} .
$$

Therefore, using Lemma 1 and assumption (13) implies $p(z) \prec q(z)$ and the function $q$ is the best dominant of (13).

In particular, taking $v=0, \eta=\gamma=1$ and $q(z)=\frac{1+A z}{1+B z}$ in the above theorem, it is easy to check that the inequality (10) holds whenever $-1 \leq B<A \leq 1$. Hence, we deduce the following corollary:

Corollary 2. Let $-1 \leq B<A \leq 1$ and $\mu \in \mathbb{C}^{*}$. Let $f \in \Sigma$ satisfy the conditions (4) and

$$
\frac{-2 z f^{\prime}(z)}{f(z)-f(-z)} \neq 0, z \in \mathbb{E} \text {. }
$$

If

$$
1+\mu\left[1-\frac{z\left(f^{\prime}(z)+f^{\prime}(-z)\right)}{f(z)-f(-z)}+\frac{z f^{\prime \prime}(z)}{f^{\prime}(z)}\right] \prec 1+\frac{(A-B) z}{(1+A z)(1+B z)}
$$

then

$$
-\left[\frac{2 z f^{\prime}(z)}{f(z)-f(-z)}\right]^{\mu} \prec \frac{(1+A z)}{(1+B z)}
$$

and the function $\frac{(1+A z)}{(1+B z)}$ is the best dominant of (16) (the power is principal one).

Using $v=0, \eta=1, \gamma=\frac{1}{a b}$ with $a, b \in \mathbb{C}^{*}, \mu=a$ and $q(z)=\frac{1}{(1-z)^{2 a b}}$ in Theorem 2, then using this result together with Lemma 3 , we deduce the following result:

Corollary 3. Let $a, b \in \mathbb{C}^{*}$ such that

$$
|2 a b-1| \leq 1 \quad \text { or } \quad|2 a b+1| \leq 1
$$

and suppose that $f \in \Sigma$ satisfy the conditions (4) and (15). If

$$
1+\frac{1}{b}\left[1-\frac{z\left(f^{\prime}(z)+f^{\prime}(-z)\right)}{f(z)-f(-z)}+\frac{z f^{\prime \prime}(z)}{f^{\prime}(z)}\right] \prec \frac{1+z}{1-z}
$$

then

$$
-\left[\frac{2 z f^{\prime}(z)}{f(z)-f(-z)}\right]^{a} \prec \frac{1}{(1-z)^{2 a b}}
$$

and the function $\frac{1}{(1-z)^{2 a b}}$ is the best dominant of (17) (the power is the principal one).

By using $v=0, \eta=\gamma=1$ and $q(z)=(1+B z)^{\frac{\mu(A-B)}{B}},-1 \leq B<A \leq 1, B \neq 0$ in Theorem 2 , then using Lemma 3 we have the next result:

Corollary 4. Let $-1 \leq B<A \leq 1$ with $B \neq 0$, and suppose that

$$
\left|\frac{\mu(A-B)}{B-1}\right| \leq 1 \quad \text { or } \quad\left|\frac{\mu(A-B)}{B+1}\right| \leq 1,
$$


where $\mu \in \mathbb{C}^{*}$. If $f \in \Sigma$ satisfy the conditions (4), (15) and

$$
1+\mu\left[1-\frac{z\left(f^{\prime}(z)+f^{\prime}(-z)\right)}{f(z)-f(-z)}+\frac{z f^{\prime \prime}(z)}{f^{\prime}(z)}\right] \prec \frac{1+[B+\mu(A-B)] z}{(1+B z)},
$$

then

$$
-\left[\frac{2 z f^{\prime}(z)}{f(z)-f(-z)}\right]^{\mu} \prec(1+B z)^{\frac{\mu(A-B)}{B}}
$$

and the function $(1+B z)^{\frac{\mu(A-B)}{B}}$ is the best dominant of (18) (the power is the principal one).

By taking $v=0, \eta=1, \gamma=\frac{e^{\iota \lambda}}{a b \cos \lambda}, a, b \in \mathbb{C}^{*},|\lambda|<\frac{\pi}{2}, \mu=a$ and $q(z)=\frac{1}{(1-z)^{2 a b e^{-\imath \lambda} \cos \lambda}}$ in Theorem 2, we obtain the following:

Corollary 5. Let $a, b \in \mathbb{C}^{*}$ and $|\lambda|<\frac{\pi}{2}$ and suppose that

$$
\left|2 a b e^{-\imath \lambda} \cos \lambda-1\right| \leq 1 \quad \text { or } \quad\left|2 a b e^{-\imath \lambda} \cos \lambda+1\right| \leq 1 .
$$

If $f \in \Sigma$ satisfy the conditions (4), (15) and

$$
1+\frac{e^{\iota \lambda}}{b \cos \lambda}\left[1-\frac{z\left(f^{\prime}(z)+f^{\prime}(-z)\right)}{f(z)-f(-z)}+\frac{z f^{\prime \prime}(z)}{f^{\prime}(z)}\right] \prec \frac{1+z}{1-z}
$$

then

$$
-\left[\frac{2 z f^{\prime}(z)}{f(z)-f(-z)}\right]^{a} \prec \frac{1}{(1-z)^{2 a b e^{-\iota \lambda} \cos \lambda}}
$$

and the function $\frac{1}{(1-z)^{2 a b e^{-l \lambda} \cos \lambda}}$ is the best dominant of (19) (the power is the principal one).

Theorem 3. Suppose that $q$ be univalent in $\mathbb{E}$ with $q(0)=1$ and $q(z) \neq 0$ for all $z \in \mathbb{E}$, such that

$$
\Re\left(1+\frac{z q^{\prime \prime}(z)}{q^{\prime}(z)}\right)>\max \left\{0 ;-\Re \frac{\delta}{\gamma}\right\}, z \in \mathbb{E},
$$

where $\gamma, \mu \in \mathbb{C}^{*}$ and $\delta, v, \eta \in \mathbb{C}$ with $v+\eta \neq 0$ and let $f \in \Sigma$ satisfy the conditions (11), (12) and

$$
\begin{gathered}
\phi(z)=-\left[\frac{2(v+\eta) z f^{\prime}(z)}{v z\left(f^{\prime}(z)+f^{\prime}(-z)\right)+\eta(f(z)-f(-z))}\right]^{\mu} . \\
{\left[\delta+\gamma \mu\left(1-\frac{v z\left[z\left(f^{\prime}(z)+f^{\prime}(-z)\right)\right]^{\prime}+\eta z\left(f^{\prime}(z)+f^{\prime}(-z)\right)}{v z\left(f^{\prime}(z)+f^{\prime}(-z)\right)+\eta(f(z)-f(-z))}+\frac{z f^{\prime \prime}(z)}{f^{\prime}(z)}\right)\right] .}
\end{gathered}
$$

If

$$
\phi(z) \prec \delta q(z)+\gamma z q^{\prime}(z),
$$

then

$$
-\left[\frac{2(v+\eta) z f^{\prime}(z)}{v z\left(f^{\prime}(z)+f^{\prime}(-z)\right)+\eta(f(z)-f(-z))}\right]^{\mu} \prec q(z)
$$

and $q$ is the best dominant of (13) (the power is the principal one).

Proof. Define a function

$$
p(z)=-\left[\frac{2(v+\eta) z f^{\prime}(z)}{v z\left(f^{\prime}(z)+f^{\prime}(-z)\right)+\eta(f(z)-f(-z))}\right]^{\mu}, z \in \mathbb{E} .
$$


From the assumptions (11) and (12) it follows that the multivalued power function $p$ has an analytic branch in $\mathbb{E}$ with $p(0)=1$ and from the (23) it follows that

$$
\mu p(z)\left[1-\frac{v z\left[z\left(f^{\prime}(z)+f^{\prime}(-z)\right)\right]^{\prime}+\eta z\left(f^{\prime}(z)+f^{\prime}(-z)\right)}{v z\left(f^{\prime}(z)+f^{\prime}(-z)\right)+\eta(f(z)-f(-z))}+\frac{z f^{\prime \prime}(z)}{f^{\prime}(z)}\right]=z p^{\prime}(z) .
$$

Consider the functions $\theta(w)=\delta w$ and $\phi(w)=\gamma$ that are analytic in $\mathbb{C}^{*}$. Also, if we let $Q(z)=z q^{\prime}(z) \phi(q(z))=$ $\gamma z q^{\prime}(z)$ and $h(z)=\theta(q(z))+Q(z)=\delta q(z)+\gamma \frac{z q^{\prime}(z)}{q(z)}$.

Since $Q(0)=0$ and $Q^{\prime}(0)=\gamma q^{\prime}(0) \neq 0$, the assumption (20) implies that $Q$ is starlike in $\mathbb{E}$ and

$$
\Re\left(\frac{z h^{\prime}(z)}{Q(z)}\right)=\Re\left(\frac{\delta}{\gamma}+1+\frac{z q^{\prime \prime}(z)}{q^{\prime}(z)}+\frac{z q^{\prime}(z)}{q(z)}\right)>0, z \in \mathbb{E} .
$$

Therefore, using Lemma 1 and assumption (22) implies $p(z) \prec q(z)$ and the function $q$ is the best dominant of (22).

Taking $q(z)=\frac{1+A z}{1+B z}$ in Theorem 3, where $-1 \leq B<A \leq 1$, according to (7) the condition (20) becomes

$$
\max \left\{0 ;-\Re \frac{\delta}{\gamma}\right\} \leq \frac{1-|B|}{1+|B|}
$$

Hence, for the particular cases $\eta=0$ and $\mu=\gamma=1$, we have the following result:

Corollary 6. Let $-1 \leq B<A \leq 1, \mu \in \mathbb{C}^{*}$ and $\delta \in \mathbb{C}$ with

$$
\max \left\{0 ;-\Re \frac{\delta}{\gamma}\right\} \leq \frac{1-|B|}{1+|B|}
$$

If $f \in \Sigma$ satisfy the conditions (4), (15) and

$$
-\left[\frac{2 f^{\prime}(z)}{z\left(f^{\prime}(z)+f^{\prime}(-z)\right)}\right]^{\mu}\left[\delta+\mu\left(1-\frac{\left(z\left(f^{\prime}(z)+f^{\prime}(-z)\right)\right)^{\prime}}{\left(f^{\prime}(z)+f^{\prime}(-z)\right)}\right)\right] \prec \delta \frac{1+A z}{1+B z}+\frac{(A-B) z}{(1+B z)^{2}},
$$

then

$$
-\left[\frac{2 f^{\prime}(z)}{f^{\prime}(z)+f^{\prime}(-z)}\right]^{\mu} \prec \frac{1+A z}{1+B z}
$$

and the function $\frac{1+A z}{1+B z}$ is the best dominant of (24) (all the powers are principal ones).

Taking $\eta=\gamma=1, \mu=0$ and $q(z)=\frac{1+z}{1-z}$ in Theorem 3, we get the following corollary:

Corollary 7. Let $\mu \in \mathbb{C}^{*}$ and $\delta \in \mathbb{C}$ with $\Re \delta \geq 0$. If $f \in \Sigma$ satisfy the conditions (4), (15) and

$$
-\left[\frac{2 z f^{\prime}(z)}{f(z)-f(-z))}\right]^{\mu}\left[\delta+\mu\left(1-\frac{z\left(f^{\prime}(z)+f^{\prime}(-z)\right)}{(f(z)-f(-z))}+\frac{z f^{\prime \prime}(z)}{f^{\prime}(z)}\right)\right] \prec \delta \frac{1+z}{1-z}+\frac{2 z}{(1-z)^{2}},
$$

then

$$
-\left[\frac{2 f^{\prime}(z)}{f^{\prime}(z)+f^{\prime}(-z)}\right]^{\mu} \prec \frac{1+z}{1-z}
$$

and the function $\frac{1+z}{1-z}$ is the best dominant of (25) (all the powers are principal ones). 


\section{Superordination and sandwich theorems}

Theorem 4. Let $q$ be convex in $\mathbb{E}$ with $q(0)=1$ and $\lambda \in \mathbb{C}$ with $\Re \lambda>0$. Let $f \in \Sigma$ satisfy the condition (4) such that $-\frac{2 z f^{\prime}(z)}{f(z)-f(-z)} \in \mathbb{Q}$ and suppose that the function

$$
\frac{-2(1+\lambda) z f^{\prime}(z)}{f(z)-f(-z)}+\frac{2 \lambda z^{2} f^{\prime}(z)\left(f^{\prime}(z)+f^{\prime}(-z)\right)}{(f(z)-f(-z))^{2}}-\frac{2 \lambda z^{2} f^{\prime \prime}(z)}{f(z)-f(-z)}
$$

is univalent in $\mathbb{E}$. If

$$
q(z)+\lambda z q^{\prime}(z) \prec \frac{-2(1+\lambda) z f^{\prime}(z)}{f(z)-f(-z)}+\frac{2 \lambda z^{2} f^{\prime}(z)\left(f^{\prime}(z)+f^{\prime}(-z)\right)}{(f(z)-f(-z))^{2}}-\frac{2 \lambda z^{2} f^{\prime \prime}(z)}{f(z)-f(-z)},
$$

then

$$
q(z) \prec-\frac{2 z f^{\prime}(z)}{f(z)-f(-z)}
$$

and $q$ is the best subordinant of (26).

Proof. Setting

$$
p(z)=\frac{-2 z f^{\prime}(z)}{f(z)-f(-z)}, z \in \mathbb{E},
$$

then $p$ is analytic in $\mathbb{E}$ with $p(0)=1$. Taking logarithmic differentiation of the above relation with respect to $z$, we have

$$
\frac{z p^{\prime}(z)}{p(z)}=1-z\left(\frac{f^{\prime}(z)+f^{\prime}(-z)}{f(z)-f(-z)}-\frac{f^{\prime \prime}(z)}{f^{\prime}(z)}\right)
$$

and a simple calculation yields that the assumption (26) is equivalent to

$$
q(z)+\lambda z q^{\prime}(z) \prec p(z)+\lambda z p^{\prime}(z) .
$$

Now, in order to prove our result we will use Lemma 2. Consider the functions $\theta(w)=w$ and $\phi(w)=\lambda$ analytic in $\mathbb{C}$ and set

$$
h(z)=z q^{\prime}(z) \phi(q(z))=\lambda z q^{\prime}(z) .
$$

Since $h(0)=0, h^{\prime}(0)=\lambda q^{\prime}(0) \neq 0$ and $q$ is convex in $\mathbb{E}$, it follows that $h$ is starlike in $\mathbb{E}$ and

$$
\Re \frac{\theta^{\prime} q(z)}{\phi(q(z))}=\Re \frac{1}{\gamma}>0, z \in \mathbb{E} .
$$

Therefore, Lemma 2 and assumption (26) imply $q(z) \prec p(z)$ and the function $q$ is the best subordinant of (26).

Taking $q(z)=\frac{1+A z}{1+B z}$ in Theorem 4 , where $-1 \leq B<A \leq 1$, we get the following corollary:

Corollary 8. Let $q$ be convex in $\mathbb{E}$ with $q(0)=1$ and $\lambda \in \mathbb{C}$ with $\Re \lambda>0$. Let $f \in \Sigma$ satisfy the condition (4) such that $-\frac{2 z f^{\prime}(z)}{f(z)-f(-z)} \in \mathbb{Q}$ and suppose that the function

$$
\frac{-2(1+\lambda) z f^{\prime}(z)}{f(z)-f(-z)}+\frac{2 \lambda z^{2} f^{\prime}(z)\left(f^{\prime}(z)+f^{\prime}(-z)\right)}{(f(z)-f(-z))^{2}}-\frac{2 \lambda z^{2} f^{\prime \prime}(z)}{f(z)-f(-z)}
$$

is univalent in $\mathbb{E}$. If

$$
\frac{1+A z}{1+B z}+\lambda \frac{(A-B) z}{(1+B z)^{2}} \prec \frac{-2(1+\lambda) z f^{\prime}(z)}{f(z)-f(-z)}+\frac{2 \lambda z^{2} f^{\prime}(z)\left(f^{\prime}(z)+f^{\prime}(-z)\right)}{(f(z)-f(-z))^{2}}-\frac{2 \lambda z^{2} f^{\prime \prime}(z)}{f(z)-f(-z)},
$$


then

$$
\frac{1+A z}{1+B z} \prec-\frac{2 z f^{\prime}(z)}{f(z)-f(-z)}
$$

and $q$ is the best subordinant of (27).

Using the same techniques as in proof of Theorem 3 and then applying Lemma 2, we could prove the next theorem:

Theorem 5. Let $\gamma, \mu \in \mathbb{C}^{*}$ and $\delta, v, \eta \in \mathbb{C}$ with $v+\eta \neq 0$ and $\Re\left(\frac{\delta}{\gamma}\right)>0$. Suppose that $q$ is convex in $\mathbb{E}$, with $q(0)=1$ and let $f \in \Sigma$ satisfy the conditions (11), (12) and

$$
-\left[\frac{2(v+\eta) z f^{\prime}(z)}{v z\left(f^{\prime}(z)+f^{\prime}(-z)\right)+\eta(f(z)-f(-z))}\right]^{\mu} \in \mathbb{Q} .
$$

If the function $\phi$ given by (21) is univalent in $\mathbb{E}$ and

$$
\delta q(z)+\gamma z q^{\prime}(z)+\sigma \prec \phi(z),
$$

then

$$
q(z) \prec-\left[\frac{2(v+\eta) z f^{\prime}(z)}{v z\left(f^{\prime}(z)+f^{\prime}(-z)\right)+\eta(f(z)-f(-z))}\right]^{\mu}
$$

and $q$ is the best subordinant of (28) (the power is the principal one).

If we combine Theorem 1 with Theorem 4 and Theorem 3 with Theorem 5, we deduce the following sandwich results, respectively:

Theorem 6. Let $q_{1}$ and $q_{2}$ be two convex functions in $\mathbb{E}$ with $q_{1}(0)=q_{2}(0)=1$ and $\lambda \in \mathbb{C}$ with $\Re \lambda>0$. Let $f \in \Sigma$ satisfy the condition (4), such that $-\frac{2 z f^{\prime}(z)}{f(z)-f(-z)} \in \mathbb{Q}$ and suppose that the function

$$
\frac{-2(1+\lambda) z f^{\prime}(z)}{f(z)-f(-z)}+\frac{2 \lambda z^{2} f^{\prime}(z)\left(f^{\prime}(z)+f^{\prime}(-z)\right)}{(f(z)-f(-z))^{2}}-\frac{2 \lambda z^{2} f^{\prime \prime}(z)}{f(z)-f(-z)}
$$

is univalent in $\mathbb{E}$. If

$$
\begin{gathered}
q_{1}(z)+\lambda z q_{1}^{\prime}(z) \prec \frac{-2(1+\lambda) z f^{\prime}(z)}{f(z)-f(-z)}+\frac{2 \lambda z^{2} f^{\prime}(z)\left(f^{\prime}(z)+f^{\prime}(-z)\right)}{(f(z)-f(-z))^{2}}-\frac{2 \lambda z^{2} f^{\prime \prime}(z)}{f(z)-f(-z)} . \\
\prec q_{2}(z)+\lambda z q_{2}^{\prime}(z),
\end{gathered}
$$

then

$$
q_{1}(z) \prec \frac{-2 z f^{\prime}(z)}{f(z)-f(-z)} \prec q_{2}(z),
$$

where $q_{1}$ and $q_{2}$ are respectively the best subordinant and the best dominant of (29).

Theorem 7. Let $q_{1}$ and $q_{2}$ be two convex functions in $\mathbb{E}$ with $q_{1}(0)=q_{2}(0)=1$ and let $\gamma, \mu \in \mathbb{C}^{*}$ and $v, \eta \in \mathbb{C}$ with $v+\eta \neq 0$ and $\Re \frac{\delta}{\gamma}>0$. Let $f \in \Sigma$ satisfy the condition (11), (12) and such that $-\frac{2 z f^{\prime}(z)}{f(z)-f(-z)} \in \mathbb{Q}$ and

$$
-\left[\frac{2(v+\eta) z f^{\prime}(z)}{v z\left(f^{\prime}(z)+f^{\prime}(-z)\right)+\eta(f(z)-f(-z))}\right]^{\mu} \in \mathbb{Q} .
$$

If the function $\phi$ given by (21) is univalent in $\mathbb{E}$ and

$$
\delta q_{1}(z)+\gamma z q_{1}^{\prime}(z) \prec \phi(z) \prec \delta q_{2}(z)+\gamma z q_{2}^{\prime}(z)
$$


then

$$
q_{1}(z) \prec-\left[\frac{2(v+\eta) z f^{\prime}(z)}{v z\left(f^{\prime}(z)+f^{\prime}(-z)\right)+\eta(f(z)-f(-z))}\right]^{\mu} \prec q_{2}(z)
$$

and $q_{1}$ and $q_{2}$ are respectively the best subordinant and the best dominant of (29) (the power is the principal one).

Author Contributions: All authors contributed equally to the writing of this paper. All authors read and approved the final manuscript.

Conflicts of Interest: "The authors declare no conflict of interest."

\section{References}

[1] Sakaguchi, K. (1959). On a certain univalent mapping. Journal of the Mathematical Society of Japan, 11(1), 72-75.

[2] Chand, R., \& Singh, P. (1979). On certain schlicht mappings. Indian Journal of Pure and Applied Mathematics, 10(9), 1167-1174.

[3] Thangamani, J. (1980). On starlike functions with respect to symmetric points. Indian Journal of Pure and Applied Mathematics, 11, 392-405.

[4] Khan, M. G., Darus, M., Ahmad, B., Murugusundaramoorthy, G., Khan, R., \& Khan, N. (2020). Meromorphic starlike functions with respect to symmetric points. International Journal of Analysis and Applications, 18(6), 1037-1047.

[5] Miller, S. S., \& Mocanu, P. T. (2000). Differential Subordinations: Theory and Applications. CRC Press.

[6] Bulboacă, T. (2002). Classes of first-order differential superordinations. Demonstratio Mathematica, 35(2), $287-292$.

[7] Royster, W. C. (1965). On the univalence of a certain integral. Michigan Mathematical Journal, 12(4), 385-387.

(C) 2021 by the authors; licensee PSRP, Lahore, Pakistan. This article is an open access article distributed under the terms and conditions of the Creative Commons Attribution (CC-BY) license (http://creativecommons.org/licenses/by/4.0/). 